\title{
NRG calculations of the ground-state energy: application to the correlation effects in the adsorption of magnetic impurities on metal surfaces
}

\author{
Rok Žitko ${ }^{1}$ \\ ${ }^{1}$ J. Stefan Institute, Jamova 39, SI-1000 Ljubljana, Slovenia
}

(Dated: December 4, 2018)

\begin{abstract}
The ground-state energy of a quantum impurity model can be calculated using the numerical renormalization group (NRG) with a modified discretization scheme with sufficient accuracy to reliably extract physical information about the system. The approach is applied to study binding of magnetic adsorbates modeled by the Anderson-Newns model for chemisorption on metal surfaces. The correlation energy is largest in the valencefluctuation regime; in the strong-coupling (Kondo) regime the Kondo-singlet formation energy is found to be only a minor contribution. As an application of the method to more difficult surface-science problems, we study the binding energy of a magnetic atom adsorbed near a step edge on a surface with strongly modulated surfacestate electron density. The zero-temperature magnetic susceptibility is determined from the field-dependence of the binding energy, thereby providing an independent result for the Kondo temperature $T_{K}$, which agrees very well with the $T_{K}$ extracted from a thermodynamic calculation.

PACS numbers: 72.15.Qm, 73.20.Hb, 68.43.-h, 05.10.Cc
\end{abstract}

The magnetism of nanoscopic objects supported on surfaces is of great current interest due to possible applications in ultra-dense data storage. The magnetic properties of adsorbates can now be studied on the single-atom level using scanning tunneling microscopes (STM) [1]. Adsorbed atoms attach to metal surfaces by forming strong (covalent) bonds in a process named chemisorption [2, 3]. The chemisorption controls the valence (and thus the magnetic moment) of magnetic adsorbates, it can lead to adsorbate-induced restructuring of surfaces, it affects superlattice growth, chemical reactions (catalysis) and other surface phenomena [3]. Using an STM, adsorbed atoms may be manipulated to form artificial nanostructures [4]. For successful manipulation of atomicscale objects it is crucial to understand the binding properties of adsorbates, i.e. to know the potential-energy surface as a function of the position of the adsorbate [2].

A highly simplified model for studying the chemisorption is the Anderson-Newns model [5, 6]: $H=H_{\text {band }}+H_{\text {imp }}+H_{c}$ with

$$
\begin{aligned}
H_{\mathrm{band}} & =\sum_{k, \sigma \in\{\uparrow, \downarrow\}} \epsilon_{k} c_{k \sigma}^{\dagger} c_{k \sigma}, \\
H_{\mathrm{imp}} & =\sum_{\sigma \in\{\uparrow, \downarrow\}} \epsilon n_{\sigma}+U n_{\uparrow} n_{\downarrow}, \\
H_{\mathrm{hyb}} & =\sum_{k, \sigma \in\{\uparrow, \downarrow\}} V_{k}\left(c_{k \sigma}^{\dagger} d_{\sigma}+d_{\sigma}^{\dagger} c_{k \sigma}\right) .
\end{aligned}
$$

$H_{\text {band }}$ describes the continuum of conduction-band electrons with dispersion $\epsilon_{k}, H_{\text {imp }}$ corresponds to an adsorbate level with energy $\epsilon$ and electron-electron repulsion $U\left(n_{\sigma}=d_{\sigma}^{\dagger} d_{\sigma}\right.$ is the level occupancy operator), while $H_{\text {hyb }}$ defines the hybridization which can be fully characterized by the function $\Gamma(\omega)=\sum_{k}\left|V_{k}\right|^{2} \delta\left(\omega-\epsilon_{k}\right)$. The adsorbate binding energy $\Delta E$ is defined as the difference between the ground state energy of the system described by the full Hamiltonian $H$ and the ground state energy of the decoupled system with $H_{\text {hyb }} \equiv 0$ (i.e. the limit where the atom is far away from the surface). While the Anderson-Newns model was originally intended to describe binding of hydrogen and alkali atoms, some properties of magnetic adsorbates can also be studied within a single-orbital approximation [7]. General binding properties can be determined qualitatively correctly using the unrestricted Hartree-Fock method [6], while the contributions due to correlations can be calculated variationally [8]. A method which could very accurately solve the problem in full generality for arbitrary energy-dependent $\Gamma(\omega)$ and for arbitrarily large interaction strength $U$ has been, however, lacking. In this work, it is shown that the binding energy can be calculated with an excellent accuracy using the numerical renormalization group (NRG) [9, 10, 11].

The NRG consists of a logarithmic discretization of $H_{\text {band }}$ into intervals $\left[\Lambda^{-(j+1)}: \Lambda^{-j}\right]$ with $\Lambda>1$, followed by a mapping to an effective one-dimensional tight-binding Hamiltonian with exponentially decreasing hopping constants $\propto$ $\Lambda^{-i / 2}$, and an iterative diagonalisation where one further site is taken into account at each step. At each iteration $i$, the calculated excitation spectrum is shifted by subtracting the lowest eigenvalue $E_{i}$ from all others. The series

$$
E_{\mathrm{NRG}}=\sum_{i=0}^{\infty} E_{i}
$$

is the ground-state energy of the effective Hamiltonian. To improve the results, several independent NRG calculations are performed for interleaved discretization meshes shifted by $\Lambda^{1-z}$ with $z \in(0: 1]$ and the final result is obtained as an average over all $z[12,13]$. To the best knowledge of the author, the quantity $E_{\mathrm{NRG}}$ has never been used to extract physical information about the system, presumably due to poor convergence properties and systematic errors of the conventional discretization scheme. These deficiencies of NRG were recently surmounted by a different discretization approach [14, 15] 
which consists of solving the differential equation

$$
\frac{d \mathcal{E}(x)}{d x}=\frac{\int_{\epsilon(x)}^{\epsilon(x+1)} \Gamma(\omega) d \omega}{\Gamma[\mathcal{E}(x)]},
$$

where the function $\mathcal{E}(x)$ with $x=j+z$ yields the discretization coefficients for each interval $j$ and each parameter $z$; the function $\epsilon(x)$ defines the discretization grid [15].

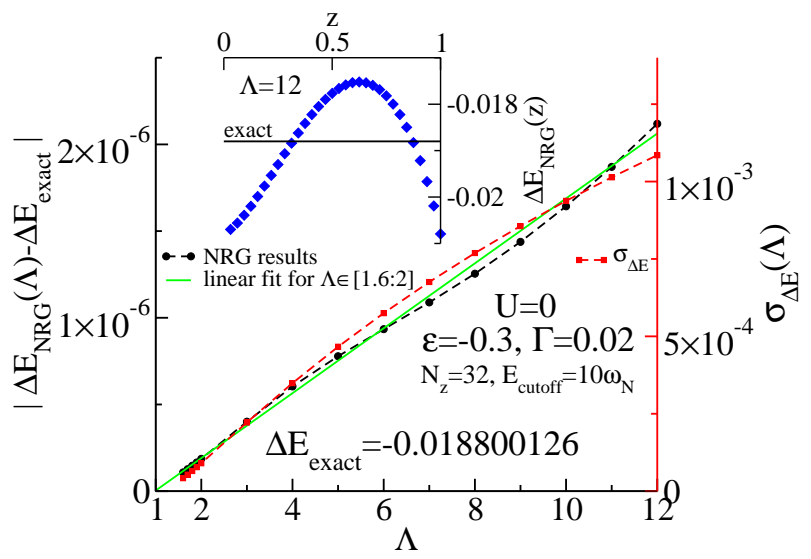

Figure 1: (Color online) $\Lambda$-dependence of the calculated binding energy of a non-interacting adsorbate. Exact binding energy $\Delta E_{\text {exact }}$ is subtracted from the numerical results $\Delta E_{\mathrm{NRG}}(\Lambda)$ (circles). Full line is a linear fit to results in the interval $\Lambda \in[1.6: 2]$. The error of the extrapolated $\Lambda \rightarrow 1$ value is $3.3 \times 10^{-9}$. The standard deviation $\sigma_{\Delta E}$ characterizes the spread of the results for different parameters $z$. An example of $\Delta E_{\mathrm{NRG}}(z)$ for $\Lambda=12$ is shown in the inset. $N_{z}=32$ different values of $z$ were used, while the parameter $E_{\text {cutoff }}=10 \omega_{N}$ defines the truncation cutoff in the NRG iteration [14].

We first consider the binding of a non-interacting adsorbate with $U=0$. In this case, the binding energy can be calculated numerically to arbitrary precision by a simple quadrature (Eq. (39) in Ref.6). For simplicity, we first consider a constant hybridization function: $\Gamma(\omega) \equiv \Gamma$ for $\omega \in[-1: 1]$ and zero otherwise. By comparing the NRG results with the exact value for a range of discretization parameters $\Lambda$, Fig. 1, we find that the binding energies are calculated with high accuracy even at $\Lambda=12$; for $\Lambda=2$ the error is $2 \times 10^{-7}$. If bare model parameters (bandwidth, $\epsilon, U$ ) are of the order of the $\mathrm{eV}$, this magnitude of the error implies that it is possible to determine the binding energy with $\mu \mathrm{eV}$ accuracy. The spread of the results $\Delta E_{\mathrm{NRG}}(z)$ for different values of $z$, as measured by the standard deviation $\sigma_{\Delta E}$ in Fig. 1, is not an indication of the error committed but rather contains physically relevant information about the effects of the hybridization. The $z$-averaging is thus an essential element of the binding energy calculation and not merely an ad-hoc procedure to accelerate the convergence.

At large $\Lambda$, the error can be decreased somewhat by increasing $N_{z}$, but the improvement is minor. A systematic improvement by one order of the magnitude can, however, be obtained by interpolation between the data points, followed by an integration over $z$ on the interval $[0: 1]$. The error is thereby reduced to $3 \times 10^{-7}$ even at $\Lambda=12$ with no additional calculations. (There is actually no need to use a uniform mesh of parameters $z$; it is more economical to choose the $z$-values as the quadrature nodes.) Using conventional discretization schemes, the errors are larger by orders of magnitude and even the extrapolated $\Lambda \rightarrow 1$ value disagrees with the exact result by $3 \times 10^{-4}$; this corresponds to an error of order $\mathrm{meV}$, which is barely acceptable especially when small effects are considered, for example in possible applications to long-range adsorbate-adsorbate interactions [16]. The use of the improved discretization scheme from Ref. 14 is thus crucial and, furthermore, the possibility of obtaining reasonably accurate results even at large $\Lambda$ implies that calculations can be performed very efficiently.

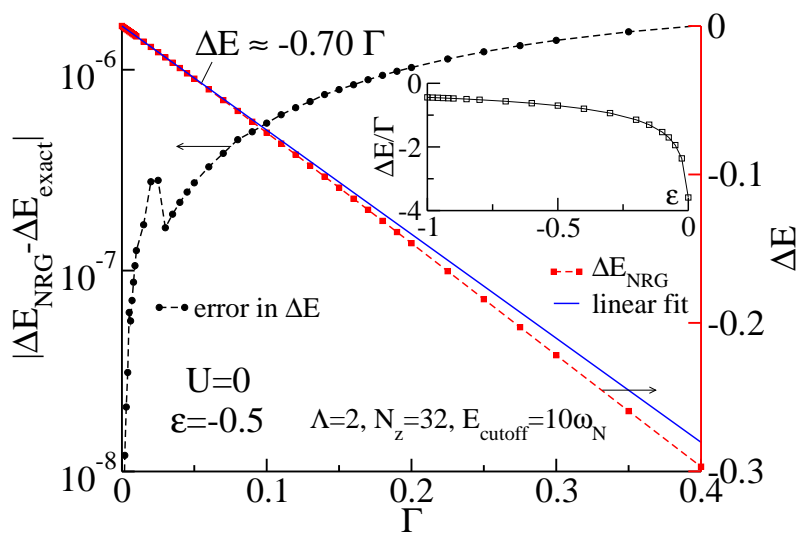

Figure 2: (Color online) Binding energy $\Delta E$ (right vertical axis) and numerical error $\Delta E_{\mathrm{NRG}}-\Delta E_{\text {exact }}$ (left vertical axis) of a noninteracting adsorbate as a function of the hybridization $\Gamma$. The proportionality coefficient $\Delta E / \Gamma=-0.70$ is extracted in the interval $\Gamma \in[0: 0.01]$. For reference, the inset shows $\Delta E / \Gamma$ as a function of $\epsilon$.

For large hybridization $\Gamma$, the adsorbate perturbs the conductance band more strongly. In NRG calculations, this is of particular concern since a finite representation of the band is used, thus finite-size effects are expected to become sizeable. We find, however, that at $\Lambda=2$ the error is bounded by $1.7 \times 10^{-6}$ for all $\Gamma$ in the interval $[0: 0.4]$, Fig. 2. The binding energy $\Delta E$ is linear in $\Gamma$ to a good approximation and the coefficient of proportionality increases in absolute value as $\epsilon$ approaches the Fermi level (see the inset in Fig. 2), where the hybridization is more effective in binding the adsorbate. The adsorbate tends to form a bond with the substrate by sharing an electron with the conductance-band states. This process is more efficient when states in the vicinity of the Fermi level are involved, since their occupancy can be inexpensively changed by the hybridization. This is similar to bond formation in twoatom molecules, where the binding energy is largest when the atomic levels are aligned.

We now study the full Anderson-Newns model with finite interaction $U$ and make comparison with the mean-field results obtained using the unrestricted Hartree-Fock (HF) method (which neglects correlation effects, see also Ref. 8). 


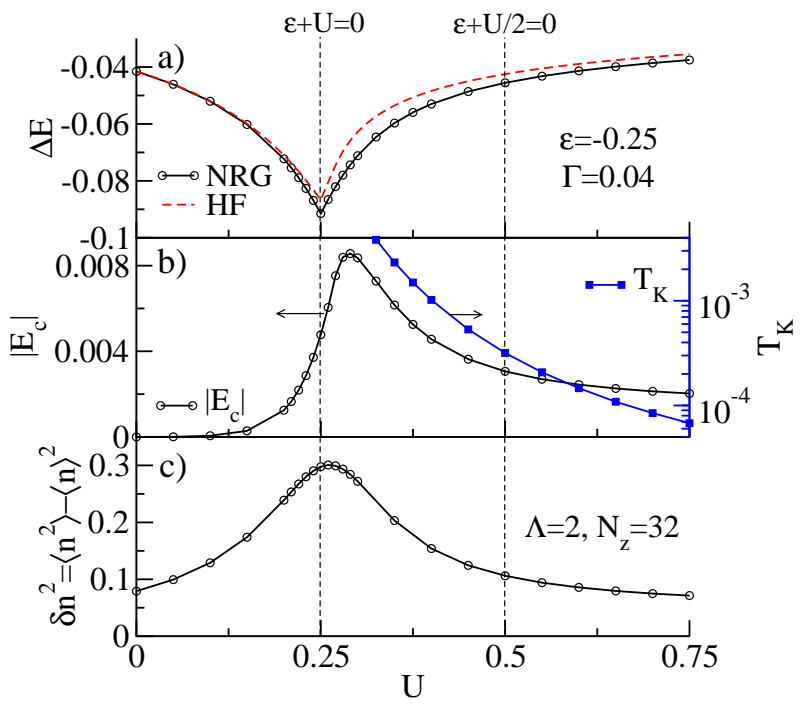

Figure 3: (Color online) a) Binding energy, b) correlation energy $E_{c}=\Delta E-\Delta E_{\mathrm{HF}}$, Kondo temperature $T_{K}$ (right vertical axis), and c) charge fluctuations of the single-impurity Anderson model as a function of the electron-electron interaction $U$. The Kondo temperature is extracted from the thermodynamic properties of the model according to Wilson's prescription $k_{B} T_{K} \chi_{\text {imp }}\left(k_{B} T_{K}\right) /\left(g \mu_{B}\right)^{2}=$ 0.07 , where $\chi_{\mathrm{imp}}$ is the impurity magnetic susceptibility [9].

The binding energy reaches its highest absolute value for $\epsilon+U=0$ when the single-particle level for an additional electron crosses the Fermi level, Fig. 3a. This behavior is similar to that of the non-interacting model: the binding energy is large when the charge fluctuates strongly. Both NRG and Hartree-Fock give the same qualitative features, but it is found that HF underestimates binding. The additional binding energy can be defined as the "correlation energy": $E_{\mathrm{c}}=$ $\Delta E-\Delta E_{H F}$. The correlation energy is largest in the valence fluctuation regime for $\epsilon+U \approx \Gamma$, see Fig. 3 b. At this point the local moment begins to form (see the decreasing charge fluctuations $\delta n^{2}$ in Fig. 3 k for increasing $U$ ) and the energy scale of magnetic correlations (the nascent Kondo regime) is the highest (see the Kondo temperature $T_{K}$ in Fig. $3 \mathrm{~b}$ ). The "Kondo-singlet formation energy" of the order of $T_{K}$ does not account for the totality of the correlation energy: it is only a small fraction, in particular in the large- $U$ limit where the Kondo temperature is strongly suppressed. The most important contribution to the correlation energy thus stems from local charge correlations, rather than from extended Kondo correlations.

The energy gain due to Kondo correlations is lost in a strong magnetic field, see Fig. 4. The quadratic reduction for low fields $\left(g \mu_{B} B \ll k_{B} T_{K}\right)$ is expected due to finite spin susceptibility at zero temperature in the strong-coupling regime [9]. From the prefactor we can extract the zero-temperature magnetic susceptibility

$$
\chi(T=0)=\frac{W\left(g \mu_{B}\right)^{2}}{4 \pi k_{B} T_{K}},
$$

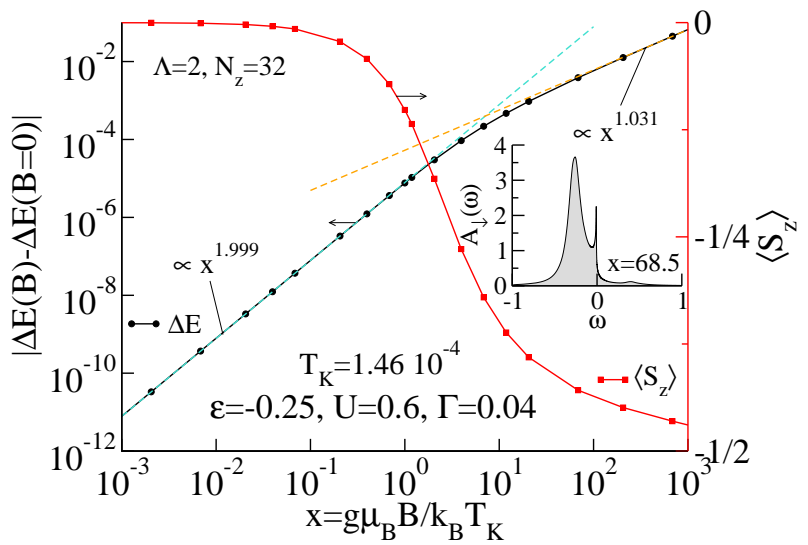

Figure 4: (Color online) Binding energy and spin polarization of a magnetic adsorbate in an external magnetic field (expressed in reduced units of $x=g \mu_{B} B / k_{B} T_{K}$ ). The inset shows the spinresolved impurity spectral function in a strong field.

where $W \approx 1.29026$ is the Wilson number [9, 17]. From $\chi(T=0)$ we then obtain a value $T_{K}^{\prime}=1.43 \times 10^{-4}$ for the Kondo temperature, which differs from the value of $T_{K}=1.46 \times 10^{-4}$ determined in a thermodynamic calculation of magnetic susceptibility by $<3 \%$. Considering that the values are obtained using entirely independent procedures, their close agreement is an exceptional confirmation of the method. The remaining small discrepancy stems mostly from the error associated with obtaining the coefficient of the $B^{2}$ contribution to the total energy in the limit of very small magnetic fields. For large fields $\left(g \mu_{B} B \gg k_{B} T_{K}\right)$, the Zeeman effect takes over and the variation of $\Delta E$ is approximately linear.

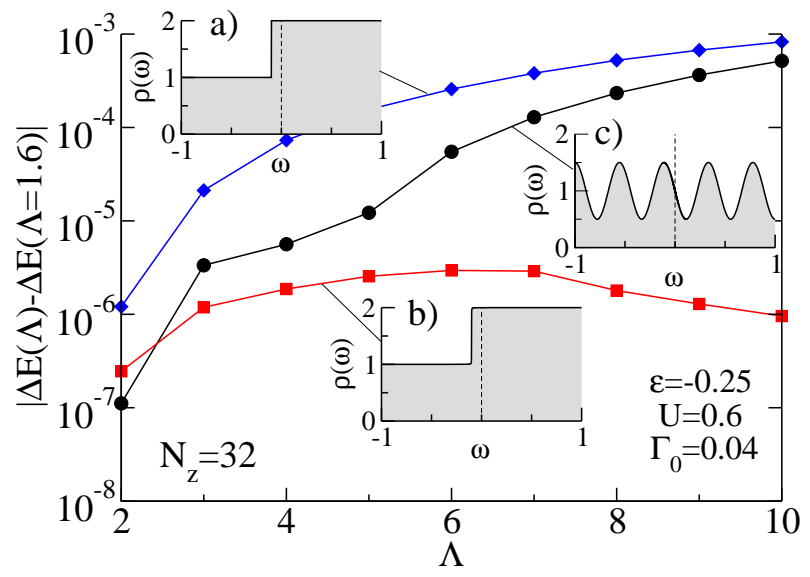

Figure 5: (Color online) $\Lambda$-dependence of the binding energy of a magnetic adsorbate hybridized to a band with energy-dependent density of states $\rho(\omega)$. The hybridization function is $\Gamma(\omega)=$ $\Gamma_{0} \rho(\omega)$ with a) sharp step function $\rho(\omega)=1+\theta\left(\omega-\omega_{0}\right)$ with $\omega_{0}=-0.1$, b) rounded step-function $\rho(\omega)=1+(1 / 2+$ $\left.(1 / \pi) \tan ^{-1}\left[\pi\left(\omega-\omega_{0}\right) / \Delta\right]\right)$, where $\Delta=0.001$, and c) oscillatory $\rho(\omega)=1+(1 / 2) \cos [(9 / 2) \pi(1+\omega)]$. The $\Lambda=1.6$ results are used as reference values and subtracted from $\Delta E(\Lambda)$. 
Albeit constant hybridization is a convenient simplification, in realistic problems $\Gamma(\omega)$ is strongly energy dependent. Three forms are considered here: sharp and rounded step functions, and an oscillatory function. The convergence with $\Lambda$ depends significantly on the form, see Fig. 5. while the error remains approximately constant at $\approx 10^{-6}$ for the rounded step function, it increases significantly for the sharpstep and oscillatory function. As expected, sharp discontinuities and variations that occur over extended energy intervals lead to larger errors than smooth localized changes.

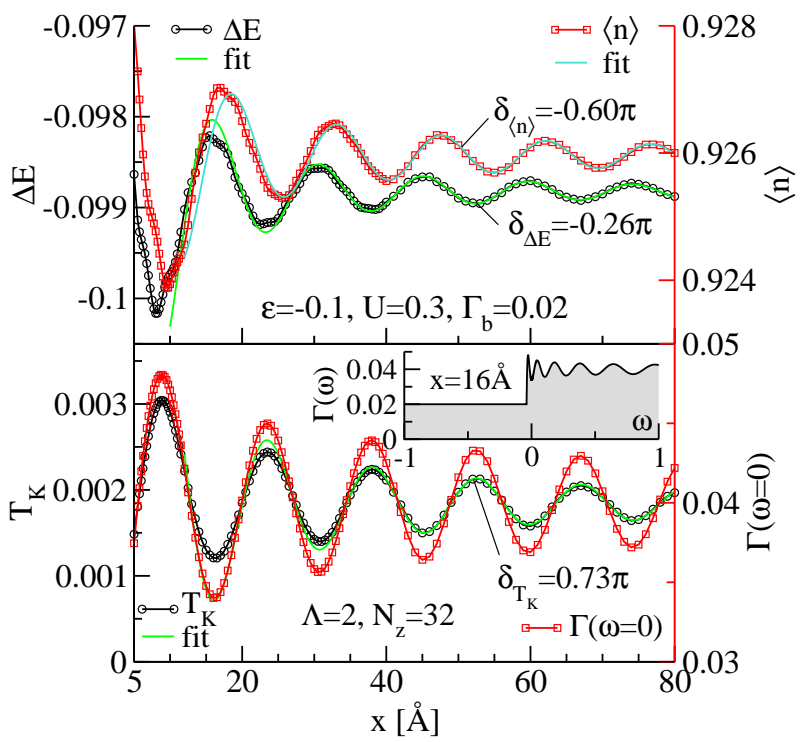

Figure 6: (Color online) Properties of a magnetic adsorbate on a surface at a distance of $x$ from a step edge modelled as a hardwall potential scatterer. Fitting by oscillatory power-law functions $A+B \cos \left(2 k_{F} x+\delta\right) / x^{\alpha}$ provides the phase shifts $\delta$ (show in the figure) and decay constants $\alpha$ : the binding energy $\Delta E$ decays as $1 / x^{3 / 2}$, the occupancy as $1 / x^{1.19}$, and the Kondo temperature as $1 / x^{1.16}$. The fitting procedure was performed with the data in the asymptotic region $x \in[40: 80] \AA$. The inset shows the hybridization function at $x=16 \AA$. $\omega_{0}=-0.039, k_{F}=0.217 \AA^{-1}$.

The capabilities of the method for problems with strongly energy-dependent hybridization are demonstrated with the example of a magnetic adsorbate in the vicinity of a step edge on a surface supporting a surface-state band. The adsorbate hybridizes both with the bulk states via $\Gamma_{b}$ (which will be assumed not to vary with energy) and with surface states via $\Gamma_{s}$. On a clean flat surface, $\Gamma(\omega)=\Gamma_{b}+\Gamma_{s} \theta\left(\omega-\omega_{0}\right)$, where $\omega_{0}$ is the onset of the surface-state band. More interesting situation occurs when the adsorbate is adsorbed near a step edge, where the local density of states of surfacestate electrons is modulated by standing waves. Modelling the step edge as a hard-wall potential, the energy-resolved charge density is $\delta n(x, \omega) \propto 1-J_{0}[2 k(\omega) x]$, where $J_{0}$ is the Bessel function, $k(\omega)$ the wave-number at energy $\omega$ and $x$ the distance from the step edge. Modelling the surface-state electrons as free electrons with effective mass $m^{*}$ we have $k(\omega)=\left[\left(2 m^{*} / \hbar\right)\left(\omega-\omega_{0}\right)\right]^{1 / 2}$. The hybridization function is thus

$$
\Gamma(\omega)=\Gamma_{b}+\Gamma_{s} \theta\left(\omega-\omega_{0}\right)\left\{1-J_{0}[2 k(\omega) x]\right\} .
$$

While it is by now established that for magnetic impurities on noble-metal surfaces $\Gamma_{s} \ll \Gamma_{b}$ [18, 19], we will nevertheless take a greatly exaggerated ratio $\Gamma_{s} / \Gamma_{b}=1$ to accentuate the effect of the energy-dependence of $\Gamma(\omega)$. In fact, on surfaces with giant Friedel oscillations [20] such ratio might be realistic.

The adsorbate properties reflect the oscillatory features in $\Gamma(\omega)$, see Fig. 6. The Kondo temperature is strongly correlated with the variation of $\Gamma$ at the Fermi level and it can be well described by a cosine function with constant phase shift $\delta_{T_{K}}$ multiplied by some envelope function which is, to a good approximation, a power-law decay $1 / x^{1.16}$. The binding energy, however, exhibits some additional structure, in particular for low values of $x$. (This is not a numerical artefact: the same result is obtained for other choices of NRG parameters.) Qualitatively similar features are visible in the adsorbate level occupancy $\langle n\rangle$, but at shifted positions $x$. The origin of these effects is thus in the details of the energy dependence of $\Gamma(\omega)$ over a wide energy interval (i.e. on the atomic scale of $\epsilon$ and $U$ ). This is unlike the Kondo temperature, which depends mostly on the values of $\Gamma(\omega)$ in the narrow interval on the scale of $T_{K}$ itself and therefore simply follows the variation of $\Gamma(\omega=0)$. It may be noted that strong binding corresponds to high Kondo temperature and that variations of $\Delta E$ and $T_{K}$ are of the same order of magnitude, pointing to a large effect of magnetic correlation in this situation.

The NRG method is a very capable tool for studying correlation effects in magnetic adsorbates on surfaces. The demonstrated favorable scaling of errors with $\Lambda$ brings more realistic (multi-orbital) models within the reach of modern computing facilities. The technique for calculating ground-state energies is very general and it can be, for example, applied to calculate the response of the system (expectation values, susceptibilities) with respect to arbitrary perturbations.

[1] A. J. Heinrich, J. A. Gupta, C. P. Lutz, and D. M. Eigler, Science 306, 466 (2004).

[2] J. K. Nørskov, Rep. Prog. Phys. 53, 1253 (1990).

[3] G. P. Brivio and M. I. Trioni, Rev. Mod. Phys. 71, 231 (1999).

[4] M. F. Crommie, C. P. Lutz, and D. M. Eigler, Science 262, 218 (1993).

[5] P. W. Anderson, Phys. Rev. 124, 41 (1961).

[6] D. M. Newns, Phys. Rev. 178, 1123 (1969).

[7] O. Ujsaghy, J. Kroha, L. Szunyogh, and A. Zawadowski, Phys. Rev. Lett. 85, 2557 (2000).

[8] K. Schönhammer, Phys. Rev. B 13, 4336 (1976).

[9] K. G. Wilson, Rev. Mod. Phys. 47, 773 (1975).

[10] H. R. Krishna-murthy, J. W. Wilkins, and K. G. Wilson, Phys. Rev. B 21, 1003 (1980).

[11] R. Bulla, T. Costi, and T. Pruschke, Rev. Mod. Phys. 80, 395 (2008).

[12] H. O. Frota and L. N. Oliveira, Phys. Rev. B 33, 7871 (1986). 
[13] V. L. Campo and L. N. Oliveira, Phys. Rev. B 72, 104432 (2005).

[14] R. Žitko and T. Pruschke, Phys. Rev. B 79, 085106 (2009).

[15] R. Žitko, Comp. Phys. Comm. (2009).

[16] K. H. Lau and W. Kohn, Surf. Sci. 75, 69 (1978).

[17] N. Andrei, K. Furuya, and J. H. Lowenstein, Rev. Mod. Phys. 55, 331 (1983).
[18] C.-Y. Lin, A. H. C. Neto, and B. A. Jones, Phys. Rev. Lett. 97, 156102 (2006).

[19] J. Henzl and K. Morgenstern, Phys. Rev. Lett. 98, 266601 (2007).

[20] P. T. Sprunger, L. Petersen, E. W. Plummer, E. Laegsgaard, and F. Besenbacher, Science 275, 1764 (1997). 